archives-ouvertes

\title{
Qualitative pivot damage analysis in aluminum printed pantographic sheets: Numerics and experiments
}

\author{
M. Spagnuolo, K. Barcz, A. Pfaff, F. Dell'Isola, P. Franciosi
}

\section{To cite this version:}

M. Spagnuolo, K. Barcz, A. Pfaff, F. Dell'Isola, P. Franciosi. Qualitative pivot damage analysis in aluminum printed pantographic sheets: Numerics and experiments. Mechanics Research Communications, Elsevier, 2017, 83, pp.47-52. <hal-01529257>

\section{HAL Id: hal-01529257 \\ https://hal.archives-ouvertes.fr/hal-01529257}

Submitted on 30 May 2017

HAL is a multi-disciplinary open access archive for the deposit and dissemination of scientific research documents, whether they are published or not. The documents may come from teaching and research institutions in France or abroad, or from public or private research centers.
L'archive ouverte pluridisciplinaire HAL, est destinée au dépôt et à la diffusion de documents scientifiques de niveau recherche, publiés ou non, émanant des établissements d'enseignement et de recherche français ou étrangers, des laboratoires publics ou privés. 


\title{
Qualitative pivot damage analysis in aluminum printed pantographic sheets: Numerics and experiments
}

\author{
M. Spagnuolo ${ }^{\mathrm{a},}, \mathrm{K}$, Barcz $^{\mathrm{b}}$, A. Pfaff ${ }^{\mathrm{c}}$, F. dell'Isola ${ }^{\mathrm{d}, \mathrm{e}}$, P. Franciosi $^{\mathrm{a}}$ \\ * CNRS, LSPM UPR3407, Lniversit' Paris 13, Sorbonne Paris Cite, 93430 Villetaneuse, France \\ ${ }^{b}$ institute of Mechanics and Printing. Warsaw University of Tectunology. Warsaw, Poland \\ 'Fraumhofer EMI, Additive Design E Manufacturing Freiburg, Germany \\ "Università di Roma "Ia Sapienza", Roma, Italy \\ e MGMoCS international Center for Mathematical and Mechanics Complex Systems Italy
}

\begin{abstract}
A B S T R A C T
In this paper we present a model in which the motions of two families of beams constituting a pantographic sheet, when described with two independent placement fields, allows to correctly simulate the points of fracture onset in axial tension test. The deformation energy is postulated in function of these independent displacements as involving an additive quadratic term of deformation energy depending on the squared norm of their difference. The first performed numerical simulations establish that the most strained pivots are exactly the ones where one observes the first rupture.
\end{abstract}

\section{Introduction}

In pantographic structures, various rupture mechanisms have been successfully reproduced from using a description of the kinematics by means of a unique placement field for the pivots interconnecting the fibers [1-4]. Yet, such a description fails to capture the observed behaviour in the class of pantographic structures for which the interconnecting pivots are the weakest structural element. In the present paper we account for a theoretical, numerical and experimental investigation aimed to start the modelling of rupture phenomena for this structure class of which the 3D aluminium specimens printed at Fraunhofer Institute, Freiburg. are a representative example: the kinematics of the connecting pivots which establish the mechanical interaction between the two involved families does not follow a one-placement field description and deserves a more detailed description. Therefore we have the need to improve the model theoretically developed in [5-13] and numerically and experimentally validated in several cases [14-22].

The specimens were manufactured in AISi $10 \mathrm{Mg}$ by the powder bed based Selective Laser Melting "SLM" process. Thereby a laser beam is used to create a solid material by melting metallic powder particles incrementally in sections and layers, which

\footnotetext{
- Corresponding author.

E-mail address: mario.spagnuoloelspm.cnirs.fi (M. Spagnuolo).
}

can be processed with different strategies and distinct parameter sets. To prevent the slender pantographic structure from breaking during the process, it was necessary to change the exposure strategy from a stripe wise to a contour based strategy resulting in prolonged laser tracks. These are necessary for a thorough fusing, due to the inertia of the optical system. The process related rough surfaces as well as the small exposure cross section of the slender beams results in a partially granular structure (see Fig. 1). Future specimen can be improved by enhanced exposure strategy (e.g. multiple exposure, pre-sintering of the material) and further adaptation of machine related parameters. To avoid any distortion due to the process related residual stresses, a stress relief heat treatment $\left(2 \mathrm{~h}\right.$ at $\left.300^{\circ} \mathrm{C}\right)$ has been applied. As a result, the usually brittle SLM material behaves more ductile [23].

As a theoretical solution for formulating a predictive model, at least in the elastic range, of considered specimens we have exploited the phase co-continuity ideas developed in [24,25]. We also want to remark that co-continuity ideas have been already used in metamaterials description, for example in [26]. The spirit which we accept is the one presented in $[27,28]$ : we consider as predominant (and physically relevant) the kinematic description of considered mechanical system and the postulation of a suitable objective action functional depending on kinematical fields. Also we consider that the rupture criterion to be used in the present context must be formulated in terms of kinematical quantities and. in particular, we conjecture that the relative displacement of the 
two families of fibers at each interconnecting pivot is the physical quantity controlling the observed rupture phenomena.

For so doing, we have modified the presented model by considering a more general description similar to the one adopted in $[29,30]$ in the case of shells and plates.

As far as plasticity does not enter in the picture, we postulate that there exists a threshold in the fibers relative displacements beyond which the interconnecting pivot breaks out. Beyond this threshold the elastic model which we use must be updated to account for the irreversible transformation to which it was submitted. The case involving plasticity becomes more complicated since a plasticity threshold will also need to be accounted for, the yield stress being possibly attained before the first fracture. In a forthcoming work the whole rupture evolution process will be studied in a quasi-static regime by introducing stiffnesses depending on the fiber relative displacement and the other deformation measures.

The presented results are preliminary but promising: they motivate the development of more sophisticated theoretical and numerical methods.

\section{Deformation energies for biphasic co-continuous pantographic structures}

Consider a reference configuration of an orthogonal pantographic lattice. It is constituted by two families of mutually orthogonal fibers which intersect one by one in a bidimensional array of regularly distributed material points. These points are modelling some cylinders with fixed dimensions, called pivots. Let be $\left\{D_{1}, D_{2}\right\}$ an orthogonal basis for the reference configuration. So we will refer to the reference configurations of the two families of fibers by using the two unitary vectors $D_{\alpha}$ with $\alpha=1,2$. In [5] it is shown how to obtain a homogenised form for the energy for this kind of structure. The authors describe a system in which the two families of fibers are described by using a single placement function $\chi$. Here we want to analyse the resistance of the pivots that interconnect the two layers of fibers to the stresses, such as to make the two layers sliding (elastically or plastically) with respect to each other. So we will have to modify the homogenised energy for the system as presented in [5] by using two independent placement functions $\chi^{\alpha}$ and introducing an interaction term in the energy as

$$
\begin{aligned}
& \mathcal{U}(\boldsymbol{\chi}(\cdot))=\int_{\Omega} \sum_{\alpha} \frac{K_{e}^{\alpha}}{2}\left(\left\|\boldsymbol{F}^{\alpha} D_{\alpha}\right\|-1\right)^{2} d \Omega \\
& +\int_{\Omega} \sum_{\alpha} \frac{K_{e}^{\alpha}}{2}\left[\frac{\nabla \boldsymbol{F}^{\alpha}\left|D_{\alpha} \otimes D_{\alpha} \cdot \nabla \boldsymbol{F}^{\alpha}\right| D_{\alpha} \otimes D_{\alpha}}{\left\|\boldsymbol{F}^{\alpha} D_{\alpha}\right\|^{2}}\right. \\
& \left.-\left(\frac{\boldsymbol{F}^{\alpha} D_{\alpha}}{\left\|\boldsymbol{F}^{\alpha} D_{\alpha}\right\|} \cdot \frac{\nabla \boldsymbol{F}^{\alpha} \mid D_{\alpha} \otimes D_{\alpha}}{\left\|\boldsymbol{F}^{\alpha} D_{\alpha}\right\|}\right)^{2}\right] d \Omega \\
& +\int_{\Omega} \frac{K_{p}}{2}\left|\arccos \left(\frac{\boldsymbol{F}^{1} D_{1}}{\left\|\boldsymbol{F}^{1} D_{1}\right\|} \cdot \frac{\boldsymbol{F}^{2} D_{2}}{\left\|\boldsymbol{F}^{2} D_{2}\right\|}\right)-\frac{\pi}{2}\right|^{\gamma} d \Omega \\
& +\int_{\Omega} \frac{K_{\mathrm{int}}}{2}\left\|\chi^{1}-\chi^{2}\right\|^{2} d \Omega .
\end{aligned}
$$

In Eq. (1) we used $\boldsymbol{F}^{\alpha}=\nabla \chi^{\alpha}$ and no sum over repeated $\alpha$ is intended. Since, exploiting [24,25], the pantographic structure can be seen as an assembly of two fiber-reinforced layers connected by the pivots, the last term of Eq. (1)

$$
\int_{\Omega} \frac{K_{\text {int }}}{2}\left\|\chi^{1}-\chi^{2}\right\|^{2} d \Omega
$$

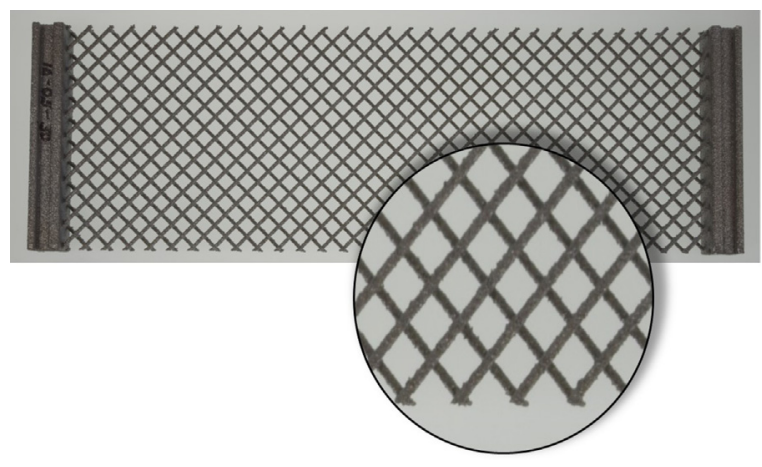

Fig. 1. 3D printing of pantographic structure with custom manufacturing parameters. Notice the granular structure, enlighted in the circle.

can be interpreted as an interaction term between two cocontinuous phases, ${ }^{1}$ described by the two placements $\chi^{\alpha}$.

Remark that the interaction in Eq. (2) is ruled by the modulus $K_{\text {int }}$ which is unknown and which in general has to be related to the flexibility of pivots. We are able to make some ansatz about the value of $K_{i n t}$, depending on the dimensions of the pivots and the material which we are considering. Infact, one can well understand that the effect of the term in Eq. (2) will be remarkable with longer pivots and it will be negligible with shorter pivots (this case is, infact, the one considered in [5]). We can consider $K_{\text {int }}$ as a modulus which either remains elastic up to the fracture threshold of the pivots or changes from elastic to elastic-plastic whenever a plastic threshold is reached before fracture. It is also possible that some microstructural damage onset at some critical threshold makes this modulus decrease before any macroscopic fracture event in the pantograph. All this will here simply be considered in terms of a possible non constancy of this modulus value, when comparing available data with performed simulations.

In this first work we will predict mainly the behaviour of pantographic structures until the first pivot rupture and we will therefore use the available experimental measure only in the regime before that point. We will not analyze in detail the possible mathematical model which describes the dependence of stiffness on deformation measures. We will present a numerical study of the behaviour of a pantographic structure modeled through a homogenised theory in which kinematics depends on the two fields, $\chi^{\mathbf{1}}$ and $\chi^{\mathbf{2}}$, which are related by the energetic term in Eq. (2). We will also show that when we choose high values for $K_{\text {int }}$ the model produces the same results of the previously introduced "1-field" model [5]. In fact, that will be the case in which interconnecting pivots do not allow for relative displacements.

In this first work, we have applied the energy modification presented in Eq. (1) to simulate the experiment reported in Figs. 2 and 3 of a tensile test applied up to fracture on an aluminium pantograph as described in Section 1. Fig. 2 reports the force-displacement curve relative to this test and Fig. 3 shows the pantograph final state with the location of the first breaks of the pivots corresponding to the maximum force on the graph.

The comparison of these experimental data with the performed simulation is presented in the next section.

Disregarding in this first work more elaborated homogenization procedures (e.g. [31-34]), we have simply applied the energy modification presented in Eq. (1) to simulate the experiment reported in

\footnotetext{
1 We could imagine also a different interaction energy between the phases, for example by adding some suitably conceived extensional springs linking the pivots of different fibers, but we refrain from doing so presently.
} 


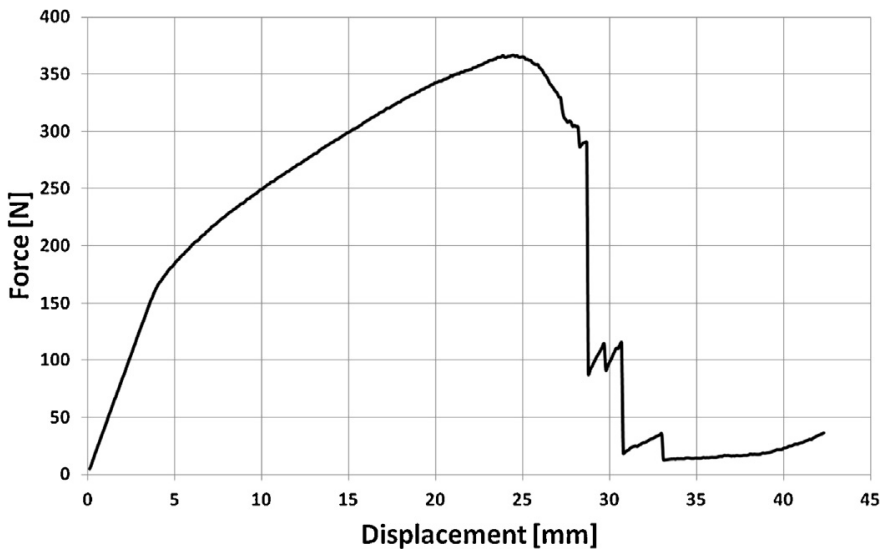

Fig. 2. Force-displacement graph of the bias extension test for the aluminum specimen shown in Fig. 1.

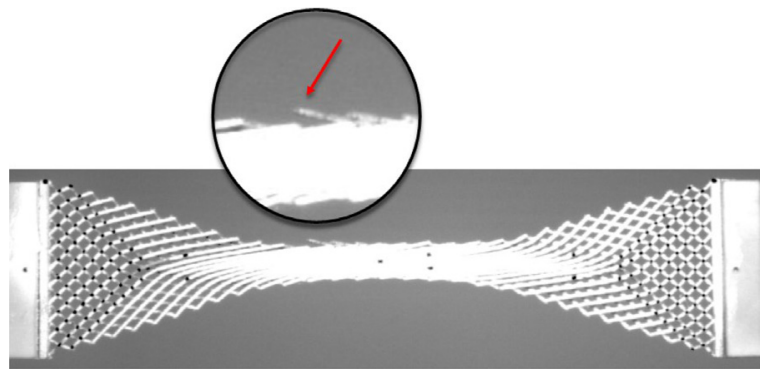

Fig. 3. Evidence of the first break in the aluminum specimen shown in Fig. 1.

Figs. 2 and 3 of a tensile test applied up to fracture on an aluminium pantograph as described in Section 1.

\section{Numerical simulations and comparisons to experiments}

Fig. 2 shows an initial linear part of the force-displacement curve, followed by a second non linear stage, of slightly decreasing slope up to the point of maximal force that closely precedes the final fracture limit. We first simulate the initial linear stage.

As far as the two families of fibers are described by using a single pivot placement function, the dominant term in the homogeneized energy in the case of a tensile test in the main direction of the pantograph is the torsion contribution, since fiber extension and bending remain of low level and effect. When attempting to match the force-displacement curve of Fig. 2 with this one-placement function, the torsion modulus is being given by the following relation [35]

$K_{p}=\frac{G_{b} \pi r^{4}}{2 h p^{2}}=6.44 \times 10^{4} \mathrm{~N} / \mathrm{m}$

where $r$ is the radius of a pivot, $h$ its height, $p$ the distance between two pivots and $G_{b}$ is the shear modulus $G_{b}=Y_{b} /[2(1+v)], Y_{b}$ and $v$ being the Young modulus and the Poisson ratio respectively. All the numerical values are listed in the following table

\begin{tabular}{lllll}
\hline$r$ & $h$ & $p$ & $Y_{b}$ & $v$ \\
\hline $0.9-1.0 \mathrm{~mm}$ & $3.0 \mathrm{~mm}$ & $4.9 \mathrm{~mm}$ & $6.8 \times 10^{9} \mathrm{~Pa}$ & 0.36
\end{tabular}

owing to the several uncertainties concerning the terms in Eq. (3) for $K_{p}$. So we could also obtain an incertitude on the $K_{p}$ value by using the incertitude on the radius of the pivot, on its height and on the distance between the pivots, which are related to the 3D printing precision.

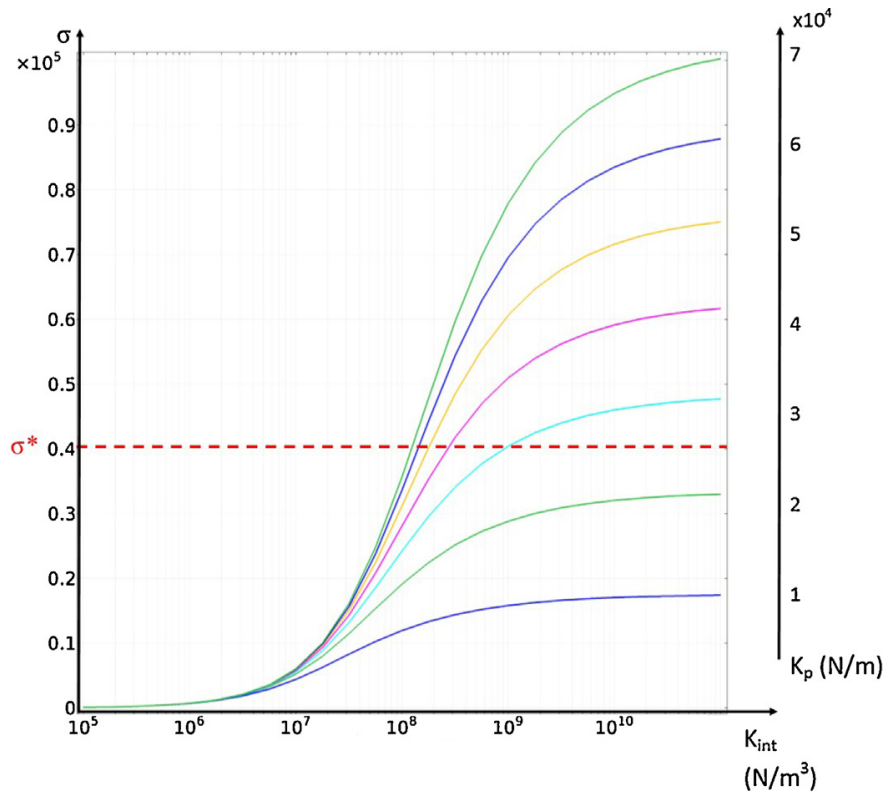

Fig. 4. Inter-dependency of the $K_{p}$ and $K_{\text {int }}$ moduli in order to always match the linear part of the experimental curve.

When the simulation makes use of the energy form in Eq. (1), related to the use of the two independent pivot placement functions, the value to be given to the interaction modulus for a match of the elastic stage is $K_{\text {int }}=1.8 \times 10^{8} \mathrm{~N} / \mathrm{m}^{3}$, when arbitrarily keeping the same value as in the "one-placement option" for the torsion modulus. The inter-dependency of these two moduli in order to always match the linear part of the experimental curve is reported in Fig. 4, showing that for both moduli there is a minimum value near which the curve slope $\sigma^{*}$ is quite insensitive to the other modulus value. These moduli are too stiff to represent the experimental behaviour during the second stage which corresponds to the onset of either some plasticity or some microstructural damage as pointed in Section 2. Yet, prior to attempt matching the force-displacement curve in this second stage, Fig. 5a and b compares the energy fields which correspond respectively to the "one-placement option" and to the "two-placement option": it is striking that the two placement option indicates (Fig. 5b) a location of maximal deformations perfectly corresponding to the experimental evidence in Fig. 3, while the one-placement option Fig. 5a corresponds with a significantly different field. It is unlikely that introducing a modulus decrease as indicated by the force-displacement curve could be capable of inverting this observation. Fig. 6 presents the obtained match up to maximal force for the $F-D$ curve in the two-placement option, with showing in Fig. 7 the used step-wise modulus decrease to obtain this match. This last figure shows how the $K_{\text {int }}$ modulus changes depending on $\delta=\left|\chi^{1}-\chi^{2}\right|$. In this mechanism of fracture there is all the physical interpretation of the presented problem: we hint that in the particular experimental measure which we analyze the fundamental fracture mechanism depends on the shear of the pivots. For well understanding this point can be useful to refer to [36]. Fig. 8 finally presents the related deformation field, where the four maxima located at the experimental fracture sites are still present. It can be interesting to look at the step-wise decrease of the modulus $K_{\text {int }}$ that we used for matching the experiment. In fact, in Fig. 9 we have the plot of this modulus along the pantographic plate for different imposed displacements and we can clearly see the arising of the effects due to the step decrease. In Fig. 9a we just find that, after an extension of about $4 \mathrm{~mm}$, the $K_{\text {int }}$ modulus has changed its value close to the four sites where we also saw the maxima 


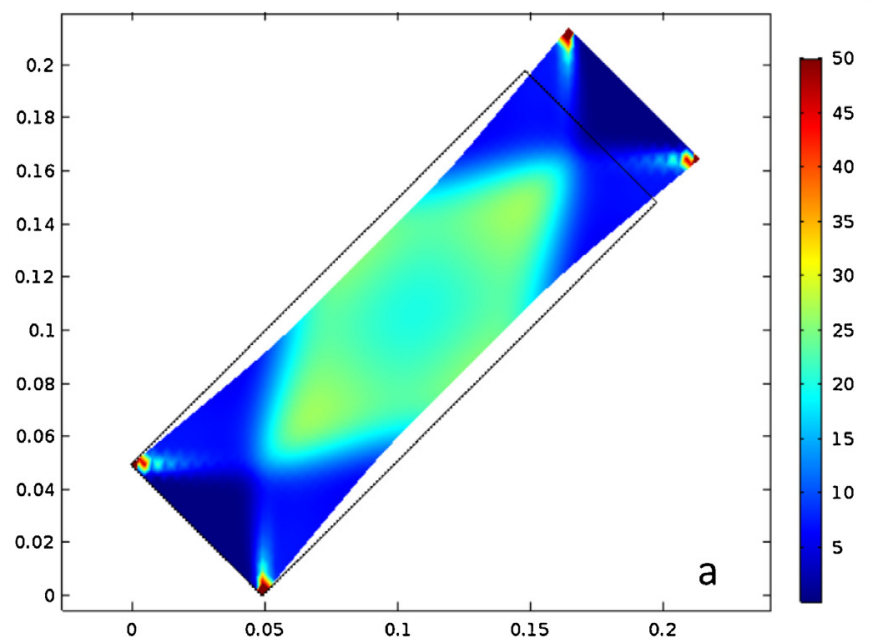

$\square$

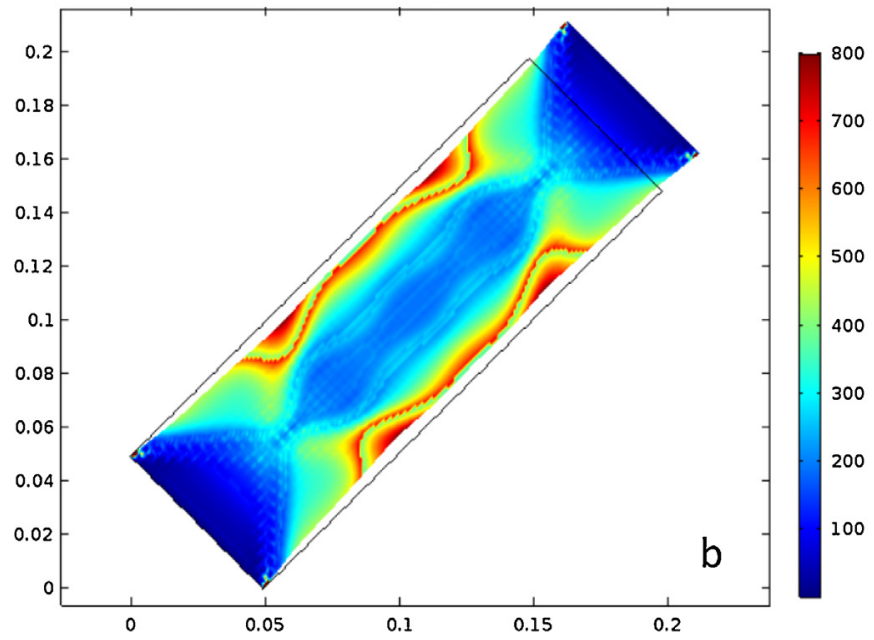

Fig. 5. The energy fields $\mathcal{U}(\mathrm{N} / \mathrm{m})$ corresponding to the "one-placement option" (a) and to the "two-placement option" (b).

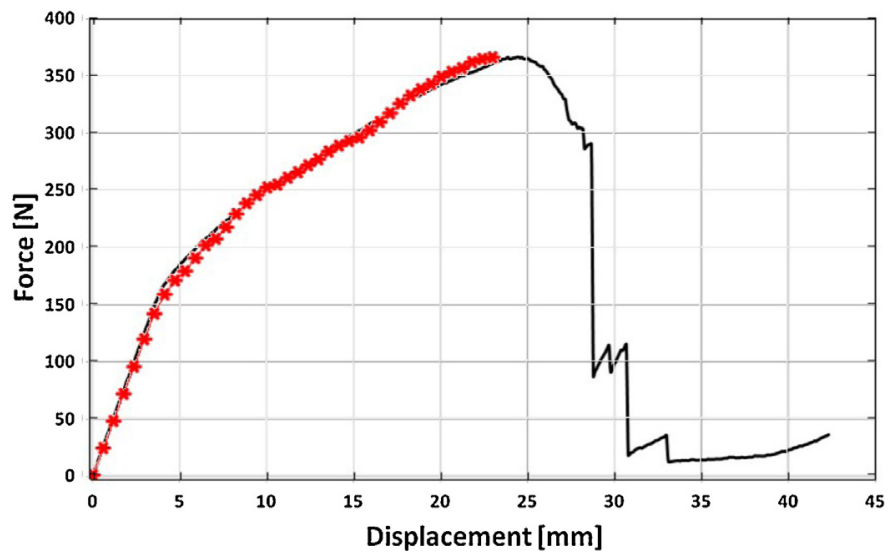

Fig. 6. The obtained match up to maximal force for the $F-D$ curve in the twoplacement option.

in Fig. 8 and which correspond to the experimental fracture sites: these four zones are the ones in which the $\delta=\left|\chi^{1}-\chi^{2}\right|$ term immediately acquires a non-zero value. In the following Fig. $9 b-d$ we see the arising of other sites in which the different thresholds in $\delta$ have been reached and consequently the $K_{\text {int }}$ modulus has changed.

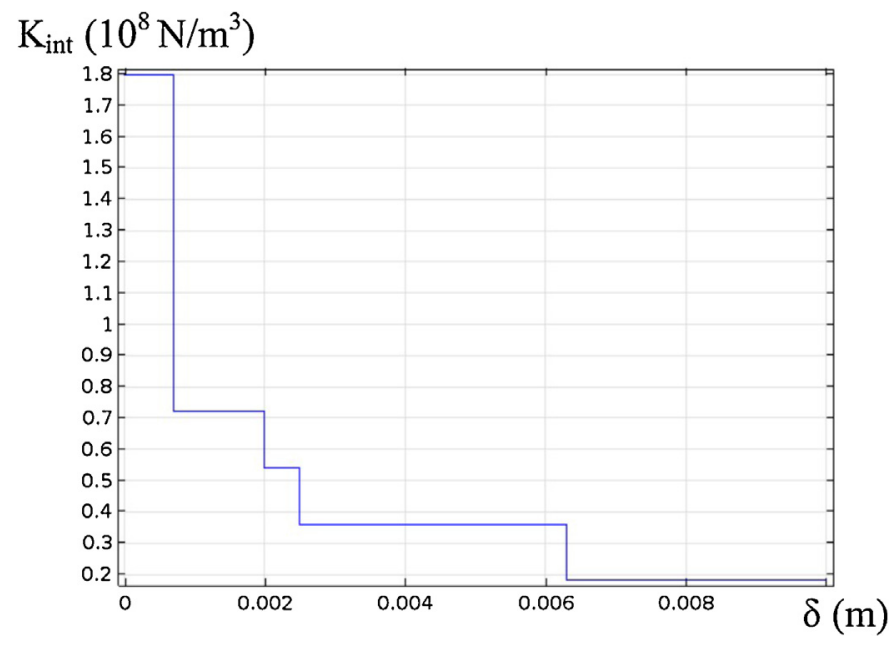

Fig. 7. Plot of the behaviour of the modulus of interaction $K_{i n t}$ in function of the difference $\delta=\left|\chi^{1}-\chi^{2}\right|$.

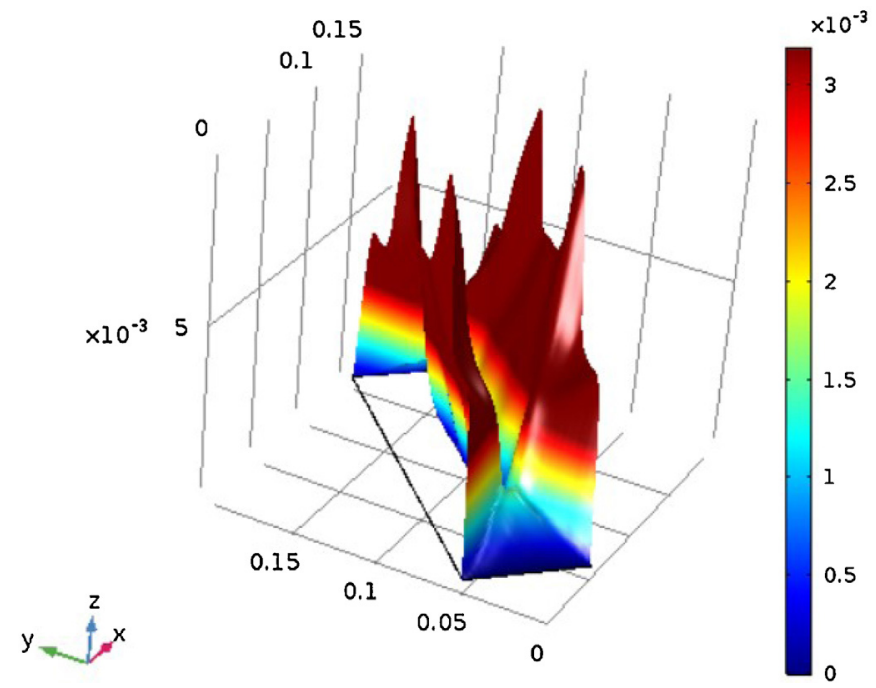

Fig. 8. Plot of the difference $\left|\chi^{1}-\chi^{2}\right|(\mathrm{m})$.

\section{Discussion}

The simple assumption that a co-continuous model assuming two different displacement fields for the two arrays of beams constituting the pantographic sheet and a simple postulation of a quadratic energy, isotropic in the difference between the two displacement fields, has allowed us to predict the main deformation features of the examined specimen up to the damage initiation. The structure of aluminum specimens at microlevel decreases remarkably the nominal resistance of constituting metal. Infact. the surface is usually very rough $(R z>80 \mu \mathrm{m})$ and therefore $0.16 \mathrm{~mm}$ of the $1 \mathrm{~mm}$ thick beam are affected by the roughness. This might be the reason why the fracture point looks granular. It is indeed not realistic to use Young and Poisson modulus for describing microscopically the involved aluminum material, which indeed most likely is even not isotropic [37]. For a discussion about the Young's modulus in anisotropic, cubic and transversely isotropic solids see [38]. This issue motivates the effort to improve from metallurgic point of view the printing process and on the other hand will require the development of more detailed and sophisticated numerical models. For well analyzing microscopic and macroscopic aspects 

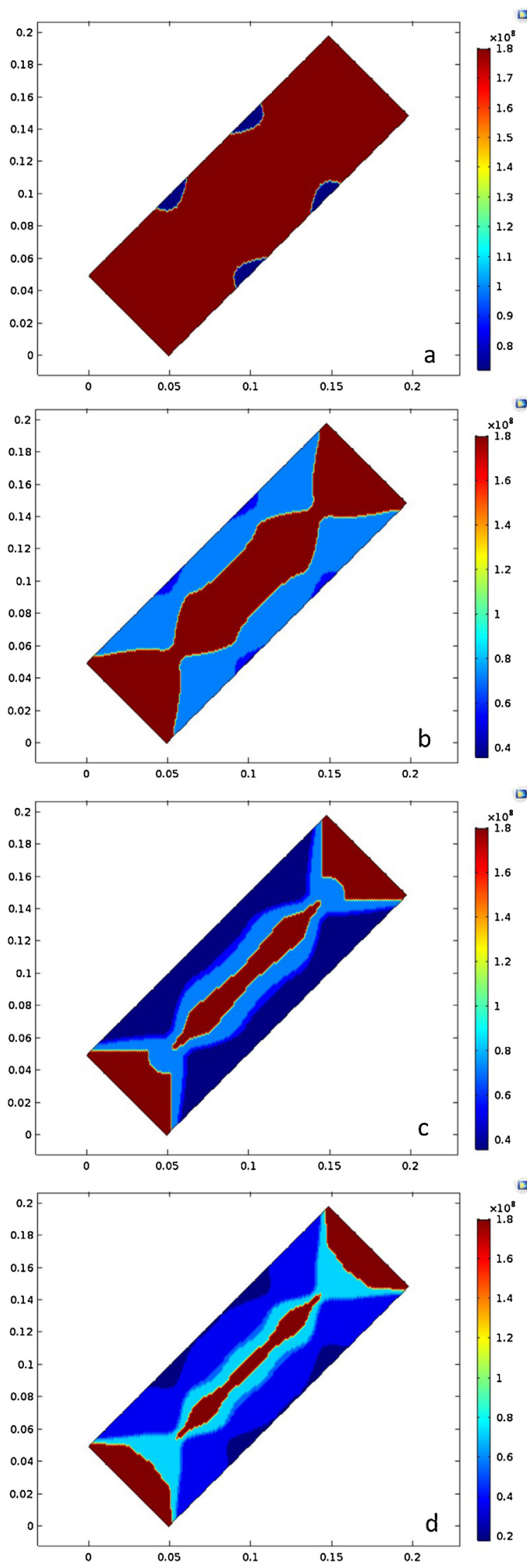

Fig. 9. Variation of the modulus $K_{\text {int }}\left(\mathrm{N} / \mathrm{m}^{3}\right)$ with following the rule on the threshold in Fig. 7.

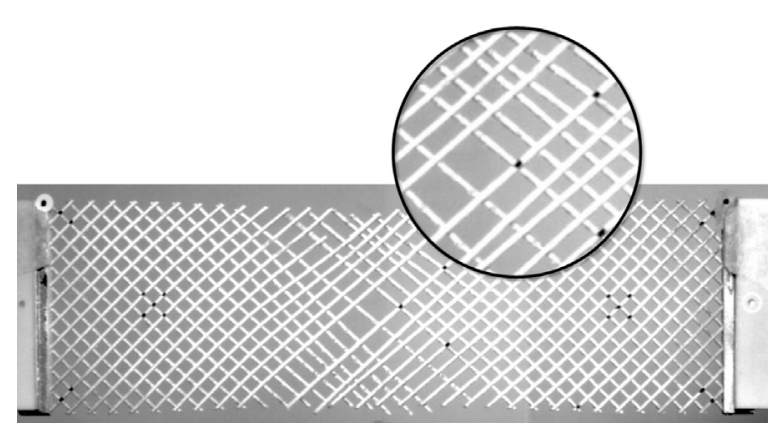

Fig. 10. Experimental evidences show that the first rupture occurs in the central pivot where most likely a brittle rupture occurs.

of the deformation and fracture mechanisms of aluminum, especially in the case of ultrafine-grained specimens, it can be useful to refer to [39]. However it is surprising that the model developed in [5] under very restrictive assumptions can be slightly improved to get, at least from a qualitative point of view, a careful prediction of the damage initiation in the standard extensional BIAS test for constructed aluminum specimens. We show (see Fig. 10) that when the aluminum specimen is too granular the rupture mechanism is not the one described in the present paper: indeed experimental evidences show that the first rupture occurs in the central pivot where most likely a brittle rupture occurs. For this rupture mechanism another rupture criterion will need to be formulated including the possibility of pivot disconnection induced by shear. To describe other typologies of rupture, for example the one occurred to the specimen shown in Fig. 10, we probably should take in account a further criterion possibly linked to the torsional angle of the pivot. Furthermore, we remark that if we tried to feat the experimental $F-D$ curve in Fig. 6 after the breaking point we probably would have to take in account also a decrease in the torsion modulus $K_{p}$.

In this work we presented the identification of the sites in which the fracture firstly arises. In a future work we can think to use the informations here obtained to perform an optimization procedure which allows to design pantographic sheets in which the characteristics of the pivots are variated to obtain a more resistent structure in the most weak sites, as for example has been done in [40-42].

\section{Conclusion}

Using 3D printing technology it has been possible to obtain some specimens of pantographic sheet using aluminum powder. The metallurgic process has not been optimized, however the first experimental evidence proves that even in the present condition the specimen has the behaviour forecast by the second gradient model developed in [5]. The observed rupture occurs for the failure of an interconnecting pivot located on the boundary of the specimen close to the last beam clamped at the short side. In the present paper we have developed a model in which the motions of two families of beams constituting a pantographic sheet are described with two independent placement fields. The deformation energy has been postulated as in [5] in function of these displacements and it has been added a further quadratic term of deformation energy depending on the squared norm of their difference. We have furthermore presented the first numerical simulations, which qualitatively confirm that the most strained pivot is exactly the one where one observes rupture. The presented results motivate further investigations both theoretical and experimental: more sophisticated printing processes and efficient numerical coding seem necessary to progress in the design of pantographic sheets. 


\section{Acknowledgements}

Mario Spagnuolo has received funding from the European Union's Horizon 2020 research and innovation programme under the Marie Skłodowska-Curie grant agreement No. 665850.

\section{References}

[1] E. Turco, F. dell'Isola, N.L. Rizzi, R. Grygoruk, W.H. Müller, C. Liebold, Fiber rupture in sheared planar pantographic sheets: numerical and experimental evidence, Mech. Res. Commun. 76 (2016) 86-90.

[2] E. Turco, N.L. Rizzi, Pantographic structures presenting statistically distributed defects: numerical investigations of the effects on deformation fields, Mech. Res. Commun. 77 (2016) 65-69.

[3] L. Placidi, A variational approach for a nonlinear 1-dimensional second gradient continuum damage model, Contin. Mech. Thermodyn. 27 (4) (2015) 623-638.

[4] A. Della Corte, A. Battista, F. dell'Isola, I. Giorgio, Modeling deformable bodies using discrete systems with centroid-based propagating interaction: fracture and crack evolution, in: Mathematical Modelling in Solid Mechanics, Springer, Singapore, 2017, pp. 59-88.

[5] F. dell'Isola, I. Giorgio, M. Pawlikowski, N. Rizzi, Large deformations of planar extensible beams and pantographic lattices: heuristic homogenization, experimental and numerical examples of equilibrium, Proc. R. Soc. A 472 (2185) (2016) 20150790, The Royal Society.

[6] F. Dos Reis, J. Ganghoffer, Equivalent mechanical properties of auxetic lattices from discrete homogenization, Comput. Mater. Sci. 51 (1) (2012) 314-321.

[7] L. Placidi, L. Greco, S. Bucci, E. Turco, N.L. Rizzi, A second gradient formulation for a 2D fabric sheet with inextensible fibres, Z. Angew. Math. Phys. 67 (5) (2016) 114.

[8] A. Della Corte, F. dell'Isola, R. Esposito, M. Pulvirenti, Equilibria of a Clamped Euler Beam (Elastica) with Distributed Load: Large Deformations, 2016, arXiv preprint arXiv: 1610.07814 .

[9] L. Placidi, E. Barchiesi, E. Turco, N.L. Rizzi, A review on 2D models for the description of pantographic fabrics, Z. Angew. Math. Phys. 67 (5) (2016) 121

[10] A. Misra, L. Placidi, D. Scerrato, A review of presentations and discussions of the workshop computational mechanics of generalized continua and applications to materials with microstructure that was held in Catania 29-31 October, 2015, in: Mathematics and Mechanics of Solids, 2016, http://dx.doi. org/10.1177/1081286516649654, 1081286516649654.

[11] L. Placidi, E. Barchiesi, A. Battista, An inverse method to get further analytica solutions for a class of metamaterials aimed to validate numerical integrations, in: Mathematical Modelling in Solid Mechanics, Springer, 2017, pp. 193-210.

[12] N. Auffray, J. Dirrenberger, G. Rosi, A complete description of bi-dimensional anisotropic strain-gradient elasticity, Int. J. Solids Struct. 69-70 (2015) 195-206.

[13] D.D. Vescovo, I. Giorgio, Dynamic problems for metamaterials: review of existing models and ideas for further research, Int. J. Eng. Sci. 80 (2014) $153-172$

[14] I. Giorgio, Numerical identification procedure between a micro-Cauchy model and a macro-second gradient model for planar pantographic structures, Z. Angew. Math. Phys. 67 (4) (2016) 95

[15] E. Turco, M. Golaszewski, A. Cazzani, N.L. Rizzi, Large deformations induced in planar pantographic sheets by loads applied on fibers: experimental validation of a discrete Lagrangian model, Mech. Res. Commun. 76 (2016) $51-56$.

[16] D. Scerrato, I. Giorgio, N.L. Rizzi, Three-dimensional instabilities of pantographic sheets with parabolic lattices: numerical investigations, $\mathrm{Z}$. Angew. Math. Phys. 67 (3) (2016) 1-19.

[17] D. Scerrato, I.A. Zhurba Eremeeva, T. Lekszycki, N.L. Rizzi, On the effect of shear stiffness on the plane deformation of linear second gradient pantographic sheets, ZAMM-J. Appl. Math. Mech./Z. Angew. Math. Mech. 96 (11) (2016) 1268-1279.

[18] L. Placidi, U. Andreaus, I. Giorgio, Identification of two-dimensional pantographic structure via a linear D4 orthotropic second gradient elastic model, J. Eng. Math. (2016) 1-21.
[19] E. Turco, M. Golaszewski, I. Giorgio, L. Placidi, Can a Hencky-Type Model Predict the Mechanical Behaviour of Pantographic Lattices? Springer Singapore, Singapore, 2017, pp. 285-311.

[20] E. Turco, M. Golaszewski, I. Giorgio, F. D’Annibale, Pantographic lattices with non-orthogonal fibres: experiments and their numerical simulations Compos. Part B: Eng. 118 (2017) 1-14.

[21] J.C. Reiher, I. Giorgio, A. Bertram, Finite-element analysis of polyhedra under point and line forces in second-strain gradient elasticity, J. Eng. Mech. (2016) 04016112.

[22] A. Della Corte, I. Giorgio, D. Scerrato, et al., Pantographic 2D sheets: discussion of some numerical investigations and potential applications, Int. J. Non-Lin. Mech. 80 (2016) 200-208.

[23] T.M. Mower, M.J. Long, Mechanical behavior of additive manufactured, powder-bed laser-fused materials, Mater. Sci. Eng.: A 651 (2016) 198-213.

[24] P. Franciosi, Laminate system schemes for effective property estimates of architectured composites with co-(dis) continuous phases, Mech. Res. Commun. 45 (2012) 70-76.

[25] P. Franciosi, A. El Omri, Effective properties of fiber and platelet systems and related phase arrangements in n-phase heterogeneous media, Mech. Res. Commun. 38 (1) (2011) 38-44.

[26] W. Lee, D.-Y. Kang, J. Song, J.H. Moon, D. Kim, Controlled unusual stiffness of mechanical metamaterials, Sci. Rep. 6 (2016).

[27] F. dell'Isola, U. Andreaus, L. Placidi, At the origins and in the vanguard of peridynamics, non-local and higher-gradient continuum mechanics: an underestimated and still topical contribution of Gabrio Piola, Math. Mech. Solids 20 (8) (2015) 887-928.

[28] G. Maugin, The method of virtual power in continuum mechanics: application to coupled fields, Acta Mech. 35 (1) (1980) 1-70.

[29] J. Altenbach, H. Altenbach, V.A. Eremeyev, On generalized Cosserat-type theories of plates and shells: a short review and bibliography, Arch. Appl. Mech. 80 (1) (2010) 73-92.

[30] H. Altenbach, V.A. Eremeyev, Large deformations of inelastic shells Key Engineering Materials, vol. 535, Trans. Tech. Publ., 2013, pp. 76-79.

[31] J.-J. Alibert, A. Della Corte, Second-gradient continua as homogenized limit of pantographic microstructured plates: a rigorous proof, Z. Angew. Math. Phys. 66 (5) (2015) 2855-2870.

[32] S. Forest, D.K. Trinh, Generalized continua and non-homogeneous boundary conditions in homogenisation methods, ZAMM-J. Appl. Math. Mech./Z Angew. Math. Mech. 91 (2) (2011) 90-109.

[33] J.-J. Alibert, A. Della Corte, I. Giorgio, A. Battista, Extensional elastica in large deformation as $\backslash$ Gamma-limit of a discrete 1D mechanical system, Z. Angew. Math. Phys. 68 (2) (2017) 42.

[34] J.-J. Alibert, A. Della Corte, P. Seppecher, Convergence of Hencky-type discrete beam model to Euler inextensible elastica in large deformation: rigorous proof, in: Mathematical Modelling in Solid Mechanics, Springer, 2017, pp. $1-12$.

[35] L. Placidi, U. Andreaus, A. Della Corte, T. Lekszycki, Gedanken experiments for the determination of two-dimensional linear second gradient elasticity coefficients, Z. Angew. Math. Phys. 66 (6) (2015) 3699-3725.

[36] U.A. Andreaus, G.C. Ruta, A review of the problem of the shear centre (s), Contin. Mech. Thermodyn. 10 (6) (1998) 369-380.

[37] D. Buchbinder, Selective Laser Melting Von Aluminiumgusslegierungen, Shaker, 2013.

[38] A. Cazzani, M. Rovati, Extrema of Young's modulus for cubic and transversely isotropic solids, Int. J. Solids Struct. 40 (7) (2003) 1713-1744.

[39] S. Billard, J. Fondere, B. Bacroix, G. Dirras, Macroscopic and microscopic aspects of the deformation and fracture mechanisms of ultrafine-grained aluminum processed by hot isostatic pressing, Acta Mater. 54 (2) (2006) $411-421$.

[40] U. Andreaus, M. Colloca, D. Iacoviello, An optimal control procedure for bone adaptation under mechanical stimulus, Control Eng. Pract. 20 (6) (2012) 575-583.

[41] U. Andreaus, M. Colloca, D. Iacoviello, Modeling of trabecular architecture as result of an optimal control procedure, in: Biomedical Imaging and Computational Modeling in Biomechanics, Springer, 2013, pp. 19-37.

[42] G. Rosi, R. Paccapeli, F. Ollivier, J. Pouget, Optimization of piezoelectric patch positioning for passive sound radiation control of plates, J. Vib. Control 19 (5) (2013) 658-673. 